\title{
Microhaematuria and Proteinuria Performance as a Measured by Urine Reagent Strips in Estimating Intensity and Prevalence of Schistosomahaematobium Infection in Nigeria
}

\author{
Akyala Is haku. A ${ }^{1, *}$, As he fo Daniel ${ }^{1}$, Tanimu Habibu' ${ }^{1}$, Ts aku Mary ${ }^{2}$, Agieni As hem Godwin ${ }^{3}$ \\ ${ }^{1}$ Department of science laboratory technology, College of Education, Akwanga \\ ${ }^{2}$ Department of biological science, College of Education, Akwanga \\ ${ }^{3}$ Dept of Microbiology,Kogi State University,Any igba
}

\begin{abstract}
Microhaematuria and proteinuria as measured by urine reagent strips are widely used to screen population at high risk of urinary schistosomiasis. This investigation was conducted to assess, if microhaematuria and proteinuria as measured by reagent strips could estimate intensity of Schistosomahaematobium infection in ende mic areas and evaluate their screening performance among children in Benue State, Nigeria. A total of 1,124 urine samples were collected, screened for microhaematuria and proteinuria using reagent strips (Combi 9) and compared to filtration technique the gold standard method. A significant correlation was observed between mic rohaematuria $(r h o=0.66, p<0.01)$, proteinuria $(r h o=0.71$, $p<0.01$ ) and intensity of Schistosomahaematobium eggs. Proteinuria had sensitivity of $95.7 \%$ and specificity of $67.7 \%$, while microhaematuria had sensitivity of $64.8 \%$ and specificity of $89.6 \%$. The proportion of false positive diagnoses was higher in proteinuria $(19.6 \%)$ than microhaematuria $(6.0 \%)$. The findings suggest that use of urine reagent strips could potentially estimate intensity of Schistosomahaematobium infection and their performance to screen urinary schistosomiasis agreed with previous observations.
\end{abstract}

Keywords Microhaematuria, Proteinuria, False Positive, Benue State, Nigeria

\section{Introduction}

Urinary schistosomiasis is a major debilitating disease caused by Schistosomahaematobium and characterized by the presence of blood in urine. Other symptoms are proteinuria, dysuria, bladder carcinoma, bladder stones, calcification of bladder wall and sometimes renal failure.

The distribution of schistosomiasis has changed over time with some countries in South America, Asia, the Caribbean and the Middle East bringing down the prevalences of the disease through a concerted public health effort[1].In sub-Saharan Africa, prevalence levels have increased and vary from one country to another; this is mostly because of water resources development, roads and dams projects, irrigationof land for agricultural purposes, inactive control programme and mostly neglect from the part of governments to implement control programmes in endemic areas.

Nigeria is one of the highly endemic countries where the

* Corresponding author:

akyalaisaac@yahoo.com (Akyala Ishaku. A)

Published online at http:/journal.sapub.org/ajmms

Copyright (C) 2012 Scientific \& Academic Publishing. All Rights Reserved disease has been unsystematically reported and large areas remain whose disease status is unknown[2].

Screening using rapid, indirect tests has been proposed as a procedure to simplify mapping surveys[3]. Haematuria (blood in urine) has been proposed as a valid indication of current infection in Schistosomahaematobium endemic populations $[2,4,5,6]$.

Testing urine with reagent strips for microhae maturia and proteinuria is such a simple and indirect diagnostic technique that could estimate the prevalence of urinary schistosomias is in school children of endemic communities. Operational research studies in Africa showed that screening using reagent strips is an effective method to identify school children requiring treatment and subsequently monitor control[ $[7,8,9]$. Several research studies reported high sensitivity and specificity of reagent strips compared to urine filtrationconsidered the most conclusive diagnostic for urinaryschistosomiasis and this procedure is expensive, cumbersome and too technical for lay use[2,7]. However, this study was undertaken to assess if microhaematuria and proteinuria as detected with reagent strips could estimate intensity of Schistosomahaematobiuminfection in endemic areas and evaluate their diagnostic performance in screening 
urinary schistosomiasis among children in Benue State, Nigeria.

\section{Materials and Methods}

\subsection{Study Area}

The study was conducted from November 2008 to September 2009 in Buruku and Katsina-Ala local government areas (LGAs) of Benue State, Nigeria which are known for their endemicity for urinary schistosomias is $[10,11]$. Before the commencement of the study, permission was sought from Directors of health and local government education authorities of both areas. The climate of the areas is tropical with two seasons, the dry season which starts from October to March and the rainy from April to October. Agricultural activities like crop farming and rearing of animals are the mainstay of the inhabitants.

\subsection{Sample Collection and Examination}

A total of 1124 urine samples were collected from pre and school children (primary and secondary) aged 3-27 years between 10:00 and 14:00 hrs using universal bottles. Urine were rapidly tested on the field using Medi Test combi 9 (Macherey-Nagel GmbH \& Co.KG, Germany) reagent strips for the determination of microhaematuria and proteinuria. Microhaematuria and proteinuria were measured as erythrocytes $/ \mu \mathrm{l}$ and $\mathrm{mg} / \mathrm{dl}$ of albumin respectively. The degree of microhaematuriaand proteinuriaconcentrations were as follows: 0 (negative), Ca.5-10 (+), Ca.50 (++) and Ca. $250(++)$ and 0 (negative), Ca.30 (+), Ca.100 (++) and Ca. $500(+++)$ respectively.

Immediately after testing with the reagent strips, $1 \mathrm{ml}$ of ordinary household bleach was added to each collected urine sample to preserve any ova present and then taken to the laboratory within $2 \mathrm{hrs}$ for paras itological exa mination. $10 \mathrm{ml}$ of urine was taken and filtered through a $12 \mu \mathrm{m}$ polycarbonate me mbrane in a filter holder. $W$ ith the help of a forceps, the filter was removed from the filter holder and placed on a slide. A drop of Lugol's iodine was added and the slide examined under microscope using $\mathrm{x} 10$ and $\mathrm{x} 40$ objective lenses. The number of eggs was counted per $10 \mathrm{ml}$ of urine and intensities of infection were classified as 1-10 eggs, 11-49 eggs and $>50$ eggs for light, moderate and heavy infections respectively.

\subsection{Statistical Analysis}

Collated data were double entered in Microsoft excel and analysed in PASW (Predictive Analysis software) version 18.0. Associations between variables were tested using Spearman correlation $(r h o)$ at $p<0.01$ significance level.

The diagnostic performance of microhaematuria and proteinuria was assessed by calculating sensitivity, specificity, positive predictive value and negative predictive value using the following formulae.

- Sensitivity $=\frac{a}{a+b}$ with

$\mathrm{a}=$ True positive

$\mathrm{b}=$ False negative

- Specificity $=\frac{c}{c+d}$

with

$\mathrm{c}=$ True negative

$\mathrm{d}=$ False positive

- Positive predictive value (PPV)

$=\frac{a}{a+d}$

- Negative predictive value (NPV)

$=\frac{c}{c+b}$

\section{Results}

Table1 shows the relationship between microhaematuria and intensity of Schistosomahaematobium eggs. Out of 151 having microhaematuria at $\mathrm{Ca} .5-10(+)$, light infection had the highest rate 62 (41.1\%); Moderate and heavy infection had $39(25.8 \%)$ and $12(7.9 \%)$ respectively. Of the 72 screened for microhaematuria at Ca.50 $(++)$, moderate infection had the highest rate with $42(58.3 \%)$ while light and heavy infection $5(6.9 \%)$ and $6(8.3 \%)$ respectively. Of the 147 screened for microhaematuria at Ca.250, heavy infection had the highest rate with $69(46.9 \%)$. It was observed that various degree of microhaematuria concentrations,,+++ and ++ corresponded to highest rateof light, moderate and heavy intensity of eggs respectively. A significant relationship was found between microhaematuria at different concentration and intensity of infection $(r h o=0.66, p<0.01)$.

The comparis on betweenmic rohaematuria as indicator of urinary schistosomiasis and the true disease status as determined by filtration technique shows that microhaematuria was detected in 370 (32.9\%), among these $302(26.9 \%)$ had both microhaematuria and presence of eggs (true positive) and 68(6.0\%) had mic rohaematuria with no presence of eggs (false negative). Of the 754 (67.1\%) screened not havingmicrohaematuria in their urine, 164 (14.6\%) had Schistosomahaematobiumeggs (false positive) and $590(52.5 \%)$ were devoid of eggs (true negative).

Table 2shows the relationship between proteinuria and intensity of Schistosomahaematobium eggs among children in Katsina-Ala and Buruku LGAs of Benue State. It was observed that of the 317 screened having proteinuria at Ca.30 $(+)$, light infection recorded the highest rate with 118 $(37.2 \%)$, while moderate in fection recorded the highest rate with $84(37.3 \%)$ out of the 225 screenedfor proteinuria at Ca.100(++). Heavy and moderate infection intensities recorded 52(43.3\%) and $54(45.0 \%)$ respectively out of the 120 screened for proteinuria at Ca.500 (+++). It was observed that various degree of proteinuria concentrations, + , ++ and +++ corresponded to highest rateof light, moderate and heavy intensity of eggs respectively although moderate infection was found having an edge over heavy infection at Ca.500. A significant spearman correlation $(r h o=0.71, p<$ 0.01 ) was found between different degrees of proteinuria 
concentrations and intensity of $S$. haematobium eggs.

The comparison between proteinuria as indicator of urinary schistosomiasis and the true disease status as determined by filtration technique shows that proteinuria was observed in 662 (58.9\%) children, 446(39.7\%) had both proteinuria and Schistosomahaematobiumeggs (true positive), while 216(19.2\%) had proteinuria with absence ofS. haematobium eggs (false positive). Of the 442 screened having no proteinuria in their urine, $20(1.8 \%)$ had S.haematobium eggs, while $442(39.3 \%)$ were devoid of $S$. haematobium eggs (true negative).

The ability of microhaematuria and proteinuria to accurately identify all those with the dis ease (sensitivity) was $64.8 \%$ and $95.7 \%$ respectively, while their ability to correctly sort out all those without the disease (specificity) was $89.7 \%$ and $67.2 \%$ respectively.Microhaematuria had higher positive predictive value (PPV) $(81.6 \%)$ than proteinuria $(67.7 \%)$, but had lower Negative Predictive Value (NPV) 78.2\% against 95.6\%.

\section{Discussion}

The present study demonstrates that the use of microhaematuria and proteinuria to estimate the intensity of urinary schis tosomias is has potential utility in discriminating intensity of infection among infected individuals in endemic areas.

The significant re lationships observed between microhae maturia, proteinuria and filtration technique clearly demonst rate that the presence or absence of microhaematuria or proteinuria in urine is function of Schistosomahaematobi um eggs excretion in urine. However, the false positive results of mic rohaematuria and proteinuria observed entails the daily variation of $S$. haematobium eggs excretion in infected individuals. The absence of microhaematuria and proteinuria in infected individuals (false negative) could be the result of new infection in which tissues of the urinary bladder and kidney have not been damaged yet.

The evaluation of only microhaematuria as indicator of urinary schistosomiasis shows sensitivity of $64.8 \%$ and specificity of $89.6 \%$. This is closely related to findings of Ugbomoikoet al.[9] who reported sensivity of $68.3 \%$ and specificity of $83.2 \%$ among school child ren of two endemic areas in southwestern, Nigeria. However, the sensitivity of microhaematuria in this study is higher than that of Anosikeet al.[2] who obtained sensitivity of $41.0 \%$ but with a similar specificity $(82.0 \%)$ in a study conducted in Bende LGA of Abia State, Nigeria. Sensitivity and specificity of microhaematuria in this study are lower than that reported among zan zibari school children in Tan zania with sensitivity of $77.0 \%$ ) and specificity of $97.0 \%[8]$. However, variation in sensitivity and specificity of microhaematuria during Schistosomahaematobiuminfection has been reported in several studies conducted in different African settings. They have been reported to vary from $41.0 \%$ to $93 \%$ and from $67 \%$ to $99 \%$ for sensitivity and specificity respectively $[2,4,6,8,9,12]$.

Table 1. Relationship between microhaematuria and intensity of Schistosomahaematobium eggs among pre and school children in Katsina-Ala and Buruku LGAs of Benue State

\begin{tabular}{|c|c|c|c|c|c|}
\hline \multicolumn{9}{|c|}{ Intensity of eggs per $10 \mathrm{ml}$ of urine } \\
\hline Microhaematuria(ery/ $\mu \mathrm{l})$ & Negative & $(1-10$ eggs $)$ & $(11-49$ eggs $)$ & $(>50$ eggs $)$ & Total \\
\hline Negative & $590(78.2)$ & $118(15.6)$ & $45(6.0)$ & $1(0.1)$ & 754 \\
\hline Ca.5-10 & $38(25.2)$ & $62(41.1)$ & $39(25.8)$ & $12(7.9)$ & 151 \\
\hline Ca.50 & $19(26.4)$ & $5(6.9)$ & $42(58.3)$ & $6(8.3)$ & 72 \\
\hline Ca.250 & $11(7.5)$ & $12(8.2)$ & $55(37.4)$ & $69(46.9)$ & 147 \\
\hline Total & $\mathbf{6 5 8}(\mathbf{5 8 . 5})$ & $\mathbf{1 9 7 ( 1 7 . 5 )}$ & $\mathbf{1 8 1}(\mathbf{1 6 . 1})$ & $\mathbf{8 8 ( 7 . 8 )}$ & $\mathbf{1 1 2 4}$ \\
\hline
\end{tabular}

Keys: $1-10$ eggs $/ 10 \mathrm{ml}$ of urine $=$ light infection

$11-49$ eggs $/ 10 \mathrm{ml}$ of urine $=$ Moderate infection

$>50$ eggs $/ 10 \mathrm{ml}$ of urine $=$ Heavy infection

Table 2. Relationship between proteinuria and intensity of Schistosomahaematobium eggs among pre and school children in Katsina-Ala and Buruku LGAs of Benue State

\begin{tabular}{|c|c|c|c|c|c|}
\hline \multicolumn{7}{|c|}{ Intensity of eggs per $10 \mathrm{ml}$ of urine } \\
\hline Proteinuria (mg/dl) & Negative & $(1-10$ eggs) & $(11-49$ eggs $)$ & $(>50$ eggs $)$ & Total \\
\hline Negative & $442(95.7)$ & $13(2.8)$ & $7(1.5)$ & $0(0.0)$ & 462 \\
\hline Ca.30 & $155(48.9)$ & $118(37.2)$ & $36(11.4)$ & $8(2.5)$ & 317 \\
\hline Ca.100 & $56(24.9)$ & $57(25.3)$ & $84(37.3)$ & $28(12.4)$ & 225 \\
\hline Ca.500 & $5(4.2)$ & $9(7.5)$ & $54(45.0)$ & $52(43.3)$ & 120 \\
\hline Total & $\mathbf{6 5 8}(\mathbf{5 8 . 5})$ & $\mathbf{1 9 7 ( 1 7 . 5 )}$ & $\mathbf{1 8 1 ( 1 6 . 1 )}$ & $\mathbf{8 8 ( 7 . 8 )}$ & $\mathbf{1 1 2 4}$ \\
\hline
\end{tabular}

Keys: $1-10$ eggs $/ 10 \mathrm{ml}$ of urine $=$ light infection

$11-49$ eggs $/ 10 \mathrm{ml}$ of urine $=$ Moderate infection

$>50$ eggs $/ 10 \mathrm{ml}$ of urine $=$ Heavy infection 
The diagnostic performance of proteinuria as indicator of urinary schistosomiasis showed sensitivity rate of $95.7 \%$ and specificity of $67.2 \%$ which are higher than findings of Brou weret al. $[13$ who obtained $65.0 \%$ and $60.0 \%$ respective ly among Zimbabwean school children. Ugbomo ikoet al.[9] reported lower sensitivity $(67.7 \%)$ and higher specificity $(79.6 \%)$ a mong school children in southwestern, Nigeria than the present study. Proteinuria is seen as a sign ofbladder damage occurring principally in severe infections. The high sensitivity $(95.7 \%)$ of proteinuria observed in this study cannot be conclusive about $S$. haematobiuminfection; this is because of the relatively high rate of false positive result $(19.2 \%)$ than that of microhaematuria $(6.0 \%)$. However, this false positive result could be due to other urinary tract infections.

The use of urine reagent strips has been proposed as an indirect method in identifying S.haematobium infected children, hence a useful tool to rapidly map the prevalence of urinary schistosomiasis in endemic areas.

The present study also shows that using microhaematuria and proteinuria as detected by reagent strips easily estimate intensity of infection; this is because they showed ability to discriminate between children at different level of infection intensity. Moreover, it was found that microhaematuria and proteinuria with higher positivity level detected infection of higher intensity (moderate and heavy).

Microhaematuria performed better in detecting moderate and heavy infection in the children than proteinuria. However, this corroboratesfindings of Anosikeet al.[2] and Ahmed[14]who found similar results among Nigerian and Yemeni children.

The positive predictive value (probability of infected children with S.haematobiumeggs among those having microhaematuria or proteinuria) was higher in children having microhaematuria $(81.6 \%)$ than proteinuria $(67.7 \%)$. This indicates that almost all children with mic rohaematuria were indeed infected with S.haematobium eggs.

In conclusion, using microhaematuria and proteinuria as detected by reagent strips is practical-cheap, fast and easy to use in estimating intensity and prevalence of S.haematobium infection. This can be used in Primary Health Care (PHC) setting with limited resources to screen and monitor $S$. haematobium infection in endemic areas.

\section{ACKNOWLEDGEMENTS}

Our sincere appreciation goes to all the children that participated in the study, their parents/guardians, teachers, head teachers and principals whose collaboration and understanding made the study feasible. We are indebted to Messrs J, Meme and C, Ansough, staff of the epidemiological units, Health Departments of Katsina-Ala and Buruku LGAs respectively for their help in translating English to local language during sample collection. We finally thank the Local Govern ment chairmen of Katsina-Ala and Buruku Areas and their respective education authorities for granting permission before commencement of the research. This paper constitutes part of a $\mathrm{PhD}$ research of the first author to the Biological Sciences Programme, AbubakarTafawaBalewa University, Bauchi, Bauchi State, Nigeria. No grant was received for this research.

\section{Conflict of Interest}

There is no any conflict of interest.

\section{REFERENCES}

[1] Bruun, B. and Aagaard-Hansen, J. The social context of schistosomiasis and its control:an introductionand annotated bibliography.UNICEF/UNDP/World Bank/WHO

[2] Special Programme for Research and Training in Tropical Diseases, 2008. 213pp.Anosike, J.C., Nwoke, B.E.B., Njoku, A.J. Validity of haematuria in the community diagnosis of urinary schistosomiasis infection. Journal of Helminthology, 2001, 75: 223-225.

[3] Brooker, S.,Beasly, M., Ndinaromtan, M., Madjiouroum, E.M., Baboguel, M., Djenguinabe, E., Hay, S.I., Bundy, D.A. Use ofremote sensing and geo graphical information systemin national helminth control programme in Chad. Bulletin of the World Health Organization 2002, 80(10):783-789.

[4] Takougang, I.,Meli, J., Fotso, S., Angwafo III, F.,Kamajeu, R., Ndumbe, P.M. Haematuria anddysuria in the self diagnosisof urinary schistosomiasis among school children in Northern Cameroon. African Journal of Health Sciences 2004,11(3\&4): 121-127.

[5] Van derWerf, M.J., De Vlas, S.J. Diagnosis of urinary schistosomiasisa novel approach to compare bladder pathology measured by ultrasound and three methods for haematuriadetection. American Journal of Tropical Medicine and Hygiene 2004,71:98-106.

[6] Robinson, E., Picon, D., Sturrock, H.J., Sabasio, A., Lado, M., Kolaczinski, J., Brooker, S. (2009). The performance of haematuria reagent strips for the rapid mapping of urinary schistosomiasis: field experience from southern Sudan. Tropical Medicine andInternational Health, 14(12): 1484-1487.

[7] Savioli,L., Renganathan, E., Montresor, A. Control of schistosomiasis, a global picture. Parasitology Today, 13: 444-448

[8] French, M.D., Rollinson, D.,Basáñez, M.G., Mgeni, A.F., Khami, I.S, Stothard, J.R.School-based control of urinary schistosomiasis on Zanzibar, Tanzania: monitoring microhaematuriawithreagent strips as a rapid urological assessment. Journal of Pediatrics Urology 2009, 3:364-368.

[9] Ugbomoiko, U.S., Dalumo, V., Ariaza, L., Bezerra, F.S.M., Heukelbach, J. A simple approach improving the performance of urine reagent strips for rapid diagnosis of urinaryschistosomiasis in Nigerian school children. MemoriasInstitutoOswaldo Cruz2009, 104(3):456-461.

[10] Amali, O. (1993). Schistosomahaematobium infection amongprimary school in Benue State, Nigeria. The Nigerian 
Journal of Parasitology 1993, 14:59-63.

[11] Houmsou, R.S., Kela, S.L., Suleiman, M.M., Ogidi, J.A. Endemicity and intensity ofvesicalschistosomiasis: epidemiologicalprofile of two Local Government Areas of Benue State-Nigeria. The Internet Journal of Health2009, 9(2).www.ispub.com.

[12] Brooker, S., Utzinger, J. Integrateddisease mapping in polyparasiticworld. GeospatialHealth 2007, 1: 141-146.
[13] Brouwer, K.C., Munatsi, A., Ndlovu, P.D., Wagatsuma, Y., Shiff, C.J.Urinary schistosomiasisin Zimbabwean school children: predictors of morbidity. African Health Sciences 2004, 4(2): 115-118.

[14] Ahmed, B.A. Two practical and cost-effective methods for urinary schistosomiasis screening in Yemeni school children. Iranian Journal of PublicHealth,38 (3): 78-83. 